\title{
EFECTOS DEL APOYO EMOCIONAL DE LAS REDES CERCANAS AL EMPRENDEDOR INDÍGENA SOBRE EL ÉXITO DE LA NUEVA EMPRESA ECOTURÍSTICA EN LA MITAD SUR DE MÉXICO
}

\author{
Virginia Barba-Sánchez* \\ Universidad de Castilla-La Mancha \\ Ericka Molina Ramírez ${ }^{* *}$ \\ Instituto Politécnico Nacional. México
}

\section{RESUMEN}

El presente trabajo pretende estudiar el impacto que el apoyo emocional proveniente de las redes cercanas ejerce en el emprendedor, a fin de determinar si estas redes constituyen un recurso de influencia relevante durante el proceso emprendedor. El contexto elegido ha sido México y los resultados obtenidos indican que el apoyo emocional resulta vital para la culminación de este proceso. Además, la creación de empresas ecoturísticas por parte de comunidades indígenas permite compatibilizar progreso económico y conservación del medio ambiente en las zonas que habitan, reduciendo su nivel de marginación social y económica.

Palabras clave: Redes sociales; creación de empresas; emprendedor social; apoyo emocional; capital Social; ecoturismo.

\section{Effects of social networks closest to the indigenous entrepreneur on new ecotourism companies' success Southern in Mexico}

\section{ABSTRACT}

The present work aims to study the impact derived from the emotional support provided from the closest networks they offer to the entrepreneur, in order to determine if this

Recibido: 9 de julio de 2014

Devuelto para su revisión: 7 de noviembre de 2014

Aceptado: 11 de diciembre de 2014

* Departamento de Administración de Empresas. Universidad de Castilla-La Mancha. Edificio Infante Don Juan Manuel. Paseo de los Estudiantes, s/n. 02006 ALBACETE (España). E-mail: Virginia.Barba@uclm.es

** Escuela Superior de Comercio y Administración (ESCA). Instituto Politécnico Nacional (IPN). Plan de San Luis, s/n. Santo Tomas. Miguel Hidalgo. 11340-CIUDAD DE MÉXICO, D.F. (México). E-mail: emolinar@ipn.mx 
networks constitute a relevant influential resource during the entrepreneurial process. The context chosen has been Mexico and the results obtained indicate that emotional support is vital for the culmination of this process. Furthermore, the creation of ecotourism companies by indigenous communities allows for compatible economic progression and conservation of the environment in zones which were habited, reducing its social and economical marginalisation level.

Keywords: Social networks; start-up businesses; social entrepreneur; emotional support; social capital; ecotourism.

\section{INTRODUCCIÓN}

A lo largo de los últimos años se ha observado un creciente interés por el estudio de la influencia que ejercen las redes sociales en el acto de emprender. Así, se ha destacado la importancia de las redes personales del emprendedor con la finalidad, entre otras, de mejorar el proceso de creación de empresas, debido a que son fuente de recursos, al poder hacer uso de los contactos para, por ejemplo, obtener información privilegiada de confianza, reducir la incertidumbre percibida, recibir soporte emocional o identificar una idea u oportunidad para iniciar un negocio. De este modo, tal y cómo estas ideas ponen de manifiesto, las redes sociales y el contexto del emprendedor representan un aspecto clave en la creación de empresas.

Desde la perspectiva teórica de la ecología de la población, se sostiene que el entorno constituye un factor clave de influencia en el proceso de creación de una empresa. Y ello debido a que, a través del mismo, las nuevas empresas pueden obtener recursos de muy diversa índole, que son de gran importancia para lograr la supervivencia, la consolidación y el crecimiento del negocio. En este contexto, es posible afirmar que las redes constituyen un factor del entorno que condiciona el proceso de creación de una empresa, contribuyendo a facilitar su culminación en función de la variedad de recursos que se encuentren en la red, de forma que existen recursos reales y/o potenciales insertados en la red de relaciones, denominados Capital Social. Sobre este particular, la literatura especializada incluye el contexto del emprendedor, afirmando que éste es susceptible de proporcionar lo que se denomina recursos soporte.

El Capital Social puede proveer beneficios tales como la reducción de los costes de transacción, la producción de bienes públicos, el suministro de información de confianza, la reducción de incertidumbre, la generación de confianza para convertir ideas en proyectos viables, la facilitación en el intercambio de recursos o la potenciación de una orientación emprendedora a través de la red. En este sentido, a pesar de que existen abundantes trabajos que han estudiado el impacto de las redes y/o el Capital Social en el proceso de creación, los trabajos que han abordado el análisis del soporte emocional que las redes sociales del emprendedor le proporcionan durante el proceso de creación de la empresa son escasos.

Por ello, es en esta última línea de investigación donde se enmarca el presente trabajo, tratando de analizar el impacto que se deriva del soporte emocional de las redes más 
próximas al emprendedor -en concreto, la familia y los amigos-, en el proceso de creación de la nueva empresa. A fin de alcanzar tal objetivo, el estudio se ha estructurado en tres apartados. El primero aborda el estudio de las redes sociales y sus recursos (e.g., el Capital Social), así como la influencia que tiene el soporte emocional, como recurso inmerso en la red, en el proceso de creación de empresas. En el segundo apartado, se describe la metodología empírica seguida, así como la población objeto de estudio, en concreto, las empresas ecoturísticas creadas por grupos indígenas en el centro y sur de México. La elección de dicha población se debe, principalmente, a la importancia que este tipo de empresas representa sobre el sustento económico, no sólo de México, sino también de otros países con problemáticas similares (e.g., índices elevados de analfabetismo, inasistencia escolar, servicios de salud inaccesibles, falta de ingresos, etc.). Países en los que se está fomentando la creación de empresas ecoturísticas como fuente de ingresos para los pueblos indígenas, lo que permite compatibilizar la rentabilización de sus tierras con la conservación de su cultura y medio ambiente, origen de oportunidades en el sector turístico. A continuación, se describen los principales hallazgos obtenidos, para, finalmente, establecer las conclusiones y las principales líneas futuras que se derivan de esta investigación.

\section{MARCO TEÓRICO E HIPÓTESIS}

\subsection{Las redes sociales y la generación de Capital Social}

En general, los agentes económicos no trabajan de forma aislada, sino que tienden a establecer interacciones económicas que se encuentran integradas en redes y estructuras sociales (Granovetter, 1985). Dichas redes constituyen un modelo en el que es posible identificar diversas estructuras sociales de relaciones humanas (Stam et al., 2014).

Teniendo en cuenta estas ideas, en el presente trabajo se considerará que la red contiene el conjunto de personas y/o grupos de empresas relacionadas, en el que se establecen lazos y acuerdos entre los miembros, y que se coordinan -por medio de los mecanismos de mercado- para llevar a cabo sus actividades.

El principal objetivo de las empresas para crear lazos entre ellas, es el mutuo apoyo que de este modo obtienen en temas relacionados con la información, el mercado y la tecnología, entre otros (Gulati et al., 2000). En este sentido, las redes sociales han sido estudiadas desde dos perspectivas principales (Anderson y Jack, 2002): (1) desde su influencia sobre los intercambios económicos y (2) desde los recursos que provee a las empresas y al proceso de creación. Es precisamente este último punto de vista el que constituye el marco de referencia de la presente investigación.

Asimismo, al hablar de los beneficios que proporcionan las redes sociales, no se debe olvidar el concepto de Capital Social. Así, Kirk et al. (2010), por ejemplo, se centran en aquellos recursos que contienen las redes sociales, y que son tan importantes que los actores llevan a cabo diversas acciones (e.g., intercambio de favores) con objeto de conseguirlos. Por tanto, cuando se usan los recursos que se encuentran en la red se conforma el denominado Capital Social (Coleman, 1998).

Sobre este particular, Stam et al. (2014) observaron que a pesar de las diferencias más o menos marcadas que puedan existir entre el concepto de redes sociales y el de Capital 
Social, es preciso tener en cuenta que el Capital Social es un recurso que se genera en las propias redes sociales y se acumula en las mismas. Desde este punto de vista, las redes sociales tienen su origen en las necesidades de relación que presentan los individuos, y que se satisfacen por medio de intercambios (Adler y Kwon, 2002).

Teniendo en cuenta la relevancia e implicaciones del concepto Capital Social en contextos tan diversos y variados, como la Sociología o la Dirección Estratégica de empresas, es posible encontrar diversas definiciones del mismo, lo que dificulta la consideración de una única definición generalizada, que se pueden agrupar bajo tres perspectivas (ídem): la externa o egocéntrica, que recoge las relaciones directas e indirectas que existen entre individuos; la interna o sociocéntrica, que se basa en la importancia de la posición relativa de un individuo en una red dada; y la neutra, que considera que el comportamiento de un actor colectivo está influenciado tanto por sus vínculos externos con otras instituciones, como por la generación de vínculos internos.

En este trabajo de investigación, dado el objeto de estudio, las definiciones de Burt (2000) y Nahapiet y Goshal, (1998) desde una perspectiva externa, son la base del mismo, pues mientras que la primera se basa en la importancia de las redes cercanas, enfatizando el hecho de que la fortaleza de esos vínculos hace posible transacciones en las que la confianza implícita posibilita que el intercambio pueda ocurrir con determinada facilidad; la segunda aporta al concepto un carácter multidimensional que, cuando las organizaciones desarrollan su actividad en contextos caracterizados por relaciones estables, que pueden plantear altos nivels de interdependencia, interacción y proximidad, logrando cotas importantes de Capital Social. Además, estas conceptualizaciones hacen referencia a un conjunto de recursos reales y potenciales que posee un grupo de individuos, de los cuales se puede disponer a través de una red de relaciones.

Considerando las redes sociales como relaciones que se crean para obtener recursos -ya sea para el propio proceso de creación de la empresa o simplemente para obtener intercambios económicos puntuales-, dichas relaciones están inmersas en una estructura social, pudiéndose clasificar en: (1) relaciones de mercado, en las cuales los productos y servicios se intercambian o se facilitan a cambio de una contraprestación monetaria; (2) relaciones jerárquicas, donde la obediencia a la autoridad se intercambia por seguridad material y espiritual; y (3) las relaciones sociales, donde se intercambian favores y obsequios (Adler y Kwon, 2002). Desde este punto de vista, el emprendedor es un factor que está influido por diversas interacciones de relaciones sociales que sin duda condicionarán el futuro de la empresa (Hormiga et al., 2007).

\subsection{Las redes sociales y su influencia en la creación de empresas}

Entre las investigaciones desarrolladas en torno al tópico de creación de empresas, se pueden encontrar enfoques de estudio centrados en las características y atributos de la personalidad del emprendedor, enfoques que estudian el propio proceso de creación de la empresa, así como aproximaciones más complejas que estudian factores sociales, culturales y económicos. En este sentido, se considera que la creación de empresas es un proceso amplio y complejo que incluye factores de muy diversa índole, como sociales, económicos, de políticas públicas, culturales y/o situacionales (Barba y Atienza, 2012). 
$\mathrm{Al}$ respecto, De Carolis y Shaparito (2006) afirman que las diferentes investigaciones que consideran las variables psicológicas, los rasgos de la personalidad y los factores demográficos como factores que caracterizan la actividad emprendedora, no son del todo fiables debido a la ambigüedad de las conclusiones obtenidas. Por este motivo, proponen la inclusión de las redes sociales como tema de investigación emergente en el ámbito de la creación de empresas. El Cuadro 1 sintetiza las principales líneas de investigación que dentro de este campo de conocimiento, abordan el tópico de las redes sociales.

\section{Cuadro 1 \\ LÍNEAS DE INVESTIGACIÓN EN CREACIÓN DE EMPRESAS Y REDES SOCIALES}

\begin{tabular}{|c|c|}
\hline Línea de investigación & Autores \\
\hline $\begin{array}{l}\text { Estudio de las redes de emprendedores y sus } \\
\text { efectos en el éxito del proceso de creación de } \\
\text { empresas }\end{array}$ & $\begin{array}{c}\text { Baron y Markman, 2003; Birley 1985; Chell } \\
\text { y Baines, 2000; Colombo y Grilli, 2005; } \\
\text { Grossman, Yli-Renko y Janakiraman, 2012; } \\
\text { Johannisson, 1996; Stam, Arzlanian y Elfring, } \\
\text { 2014; Witt, } 2004\end{array}$ \\
\hline $\begin{array}{l}\text { Análisis de las capacidades sociales para } \\
\text { crear una empresa y desarrollar redes }\end{array}$ & $\begin{array}{c}\text { Greve y Salaff, 2003; Johannisson, 1996; } \\
\text { Johannisson y Monsted, 1997; Moran, 2005; } \\
\text { Ostgaard y Birley, } 1994\end{array}$ \\
\hline $\begin{array}{l}\text { Importancia del soporte emocional } \\
\text { que proporciona la red más próxima al } \\
\text { emprendedor }\end{array}$ & $\begin{array}{l}\text { Birley, 1985; Brüderl y Preisendörfer, 1998; } \\
\text { Greve y Salaff, 2003; Hite y Hesterly, 2001; } \\
\text { Larson y Starr, 1993; Lee, Ruan y Lai, 2005; } \\
\text { Ng, 2004; Smith y Lohrke, 2008; Van der Gaag } \\
\text { y Snijders, 2005; Witt, 2004; Young y Welsch, } \\
1993 \\
\end{array}$ \\
\hline $\begin{array}{l}\text { Características, tipos y etapas de maduración } \\
\text { de las redes en empresas de nueva creación }\end{array}$ & $\begin{array}{c}\text { Birley, 1985; Butler y Hansen, 1991; Chell y } \\
\text { Baines, 2000; Donckels y Lambrecht, 1995; } \\
\text { Larson y Starr, 1993; Monsted, 1995; Szarka, } \\
1990\end{array}$ \\
\hline $\begin{array}{l}\text { La red como proveedora de recursos en el } \\
\text { proceso de creación de una nueva empresa }\end{array}$ & $\begin{array}{l}\text { Anderson y Miller, 2003; Au y Kwan, 2009; } \\
\text { Blesa y Ripollés, 2006; Bollingtoft y Ulhoi, } \\
\text { 2005; Chandler y Hanks, 1998; Chell y Baines, } \\
\text { 2000; Hoang y Antoncic, 2003; Liao y Welsch, } \\
\text { 2003; Solymossy, 2000; Tsai, Macmillan y } \\
\text { Low, 1991 }\end{array}$ \\
\hline Capital relacional y creación de empresas & $\begin{array}{c}\text { Hormiga, Batista y Sánchez, 2007; Maula, } \\
\text { Autio y Murray, 2001; Ostgaard y Birley, } 1994\end{array}$ \\
\hline
\end{tabular}

Fuente: Elaboración propia.

Como parte del proceso de creación de una empresa, existe un periodo de preparación previo al acto de emprender en el que va madurando la decisión del individuo de convertirse en emprendedor. Este periodo está basado en un complejo proceso de motivación, fundamentado, por una parte, en sus características psicológicas y demográficas y, por otra, en las 
expectativas generadas con respecto a la nueva empresa (Barba y Atienza, 2012). En dicho periodo se pueden establecer también distintas fases, que comprenden desde la identificación de la idea hasta la puesta en marcha efectiva del nuevo negocio, convirtiéndolo en un proceso dinámico de interacción entre el potencial emprendedor y su entorno.

Butler y Hansen (1991) clasifican las redes en tres tipos, de acuerdo con su etapa de desarrollo (véase Cuadro 2). En primer lugar, cabe señalar las redes sociales referidas que existen antes de la creación de la empresa (ex ante), como pueden ser la familia, los amigos, los conocidos, etc. Por medio de estas redes se puede obtener apoyo y/o información para la identificación de una idea u oportunidad para iniciar el negocio (Dubini y Aldrich, 1991). En segundo lugar, es posible destacar las redes personales que se crean una vez puesta en marcha la empresa (ex post), cuando el emprendedor establece vínculos con otros integrantes de la red con el fin de facilitar la consolidación de la misma (Coleman, 1988). Estas redes proporcionan recursos para iniciar el negocio, consolidarlo y hacerlo competitivo, incluyendo aspectos tales como la información o el intercambio de conocimiento y bienes. Por último, una vez que la empresa se ha consolidado, se pueden mencionar las redes estratégicas, que fundamentalmente se caracterizan por tener relación con los competidores, lo que permite minimizar riesgos (Gulati et al., 2000). Por tanto, las redes influyen sobre las diferentes etapas que componen el proceso de creación de empresas (Blesa y Ripollés, 2006).

\section{Cuadro 2 \\ CLASIFICACIÓN DE LAS REDES}

\begin{tabular}{|c|l|c|}
\hline \multicolumn{1}{|c|}{ Criterio de clasificación } & \multicolumn{1}{c|}{ Tipos de red } & Autores \\
\hline Etapa de desarrollo de la red & $\begin{array}{l}\text { Redes Sociales } \\
\text { Redes Personales } \\
\text { Redes Estratégicas }\end{array}$ & Butler y Hansen, 1991 \\
\hline Nivel de formalidad de la red & $\begin{array}{l}\text { Redes Formales } \\
\text { Redes Informales }\end{array}$ & $\begin{array}{c}\text { Barney, 1985; Birley, 1985; } \\
\text { Donckels y Lambrecht, 1995; } \\
\text { Becerra, 2008 }\end{array}$ \\
\hline Nivel de cercanía de las redes & $\begin{array}{l}\text { Redes Cercanas } \\
\text { Redes Ampliadas }\end{array}$ & $\begin{array}{c}\text { Dubini y Aldrich, 1991; Ng, } \\
\text { 2004; Ostgaard y Birley, 1994 }\end{array}$ \\
\hline
\end{tabular}

Fuente: Elaboración propia.

Por otra parte, considerando el nivel de formalidad de la red, se pueden distinguir las redes formales, que incluyen las agencias locales, estatales y federales, los bancos, los contables, los abogados, las cámaras de comercio, etc. (Birley, 1985), de las informales, que están constituidas, fundamentalmente, por la familia, los amigos y los compañeros de trabajo o de escuela, y que sin duda pueden informar y dar opciones al emprendedor (ídem). Desde esta perspectiva, ambas pueden representar un apoyo, tanto para el individuo que está pensando en crear la empresa, como para las empresas recién creadas (Witt, 2004), puesto que son una vía de acceso a recursos valiosos y críticos para resolver problemas (Kantis, 2004); si bien parece que el nivel de confianza que el emprendedor tenga con cada una de ellas, influye en los potenciales beneficios que generen dichas redes (Francis y Sandberg, 2000; Zahra et al., 2006). 
La familia y los amigos, como parte de esas redes informales con mayor grado de confianza con el emprendedor, juegan un rol importante en el proceso de creación de una empresa, pues conforman las redes primarias o cercanas del empresario (Lee et al., 2005), que lo acompañaran a lo largo de todo el proceso, proveyendo de un recurso necesario, según Brüderl y Preisendörfer (1998), para garantizar la finalización del mismo: el apoyo emocional o moral. Así, Hormiga et al., (2007) afirman, tras analizar 130 nuevas empresas españolas, que tanto el apoyo emocional como la ayuda activa que el emprendedor recibe de sus redes cercanas tienen una influencia positiva con su éxito.

Por su parte, Lee et al. (2005) argumentan que en entornos con una cultura colectivista, donde el bien común prima sobre el individual, el apoyo de las redes cercanas al emprendedor es básico para entender el éxito de la puesta en marcha de una empresa. Por ello, se plantea la siguiente hipótesis general de trabajo, que se desglosa para recoger el efecto de cada uno de los colectivos que conforman las redes sociales cercanas al emprendedor:

H: Las redes sociales cercanas al emprendedor proporcionan recursos de apoyo emocional para el éxito del proceso de creación de empresas ecoturísticas indígenas. H1: El apoyo emocional de la familia del emprendedor influye de forma positiva en el éxito del proceso de creación de empresas ecoturísticas indígenas.

H2: El apoyo emocional de los amigos del emprendedor influye de forma positiva en el éxito del proceso de creación de empresas ecoturísticas indígenas.

En esta línea de investigación, Tsai et al. (1991), partiendo de los presupuestos de la Teoría de la Ecología de la Población, concluyen que el entorno y la estrategia son de vital importancia para las nuevas empresas. Y ello, porque al estudiar las relaciones sociales que el empresario establece con su entorno para conseguir información, recursos tangibles y apoyo social, observan cómo el éxito o fracaso de la nueva empresa está condicionado por estos contactos.

Del mismo modo, Chandler y Hanks (1998) señalan que, entre los factores más importantes que influyen en el proceso de creación, se debe incluir el contexto en el que se desarrolla el emprendedor. Contexto que es susceptible de proveer soporte a los emprendedores, siempre y cuando éstos sean capaces de detectar las oportunidades. Bajo esta perspectiva, el emprendedor trata de entablar diversas relaciones sociales con objeto de obtener recursos, bien sea información, servicios o favores (e.g., Witt, 2004). También Larson y Starr (1993) asegura que la habilidad del empresario emprendedor para identificar, cultivar y gestionar su red social es crítica para la supervivencia y el éxito de la nueva empresa.

No obstante, algunos trabajos consideran que el éxito de las empresas de reciente creación es una variable difícil de medir debido a las características propias de los nuevos negocios, como son la escasez de beneficios en los primeros años o la ausencia de información histórica financiera, entre otros. Con el ánimo de caracterizar el crecimiento y resultados de las empresas de reciente creación, Chandler y Hanks (1998) proporcionan ejemplos de indicadores que permiten solventar algunas de las dificultades a las que se enfrentan los investigadores en este sentido, especialmente en aquellos supuestos en los que los empresarios no están dispuestos a ofrecer información al respecto. 
Witt (2004) sostiene que dependiendo del grado de desarrollo que la compañía haya alcanzado en el proceso fundacional, hay diferentes posibilidades para definir el éxito. Éxito que puede medirse tanto por indicadores objetivos como por indicadores subjetivos. Entre los primeros es posible mencionar (Chell y Baines, 2000): la persistencia en el mercado, entendida como el número de años que la empresa lleva operando en el mercado desde que inició sus actividades; las tasas de crecimiento, tanto en ventas, como en el número de empleados; y otros datos de los estados financieros. Por su parte, las medidas subjetivas pueden clasificarse en dos grandes grupos: las que hacen referencia a la satisfacción del emprendedor en relación a los resultados empresariales (Chandler y Hanks, 1993) y las que se fundamentan en la comparación con respecto a los competidores (Hormiga et al., 2007).

Ahora bien, los indicadores subjetivos no pueden sustituir a los objetivos, al basarse en expectativas o en información imperfecta del emprendedor (Brüderl y Preisndorfer, 1998), por lo que en este trabajo de investigación se opta por la elección de dos indicadores objetivos concretos del resultado empresarial: la consolidación de la empresa, en función del número de años que la empresa lleva operando en el mercado, y su crecimiento, medido en función del ratio de variación del número de empleados. La elección de esta variable para medir el crecimiento empresarial se fundamenta en la especial relevancia que la misma tiene en el contexto de estudio, en consonancia con los planteamientos de Dana (1995) y de Barba y Molina (2014): en el seno de las comunidades indígenas analizadas, la principal razón para crear la empresa ha sido la generación de empleo en la zona a fin de evitar el éxodo de sus miembros a centros urbanos y la consiguiente desintegración, e incluso desaparición, de la Comunidad. Así, las hipótesis generales anteriormente propuestas, se pueden expresar atendiendo al siguiente desglose:

H1.1: El apoyo emocional de la familia del emprendedor, influye de forma positiva en la consolidación de la nueva empresa.

H1 .2: El apoyo emocional de la familia del emprendedor, influye de forma positiva en el crecimiento de la nueva empresa.

H2.1: El apoyo emocional de los amigos del emprendedor, influye de forma positiva en la consolidación de la nueva empresa.

H2.2: El apoyo emocional de los amigos del emprendedor, influye de forma positiva en el crecimiento de la nueva empresa.

\section{METODOLOGÍA DE LA INVESTIGACIÓN}

\subsection{Población y recogida de datos}

La población objeto de estudio de esta investigación (empresas ecoturísticas creadas en el seno de comunidades indígenas de la mitad sur de México) se caracteriza por una serie de aspectos relacionados con las características particulares y problemática diferencial de los pueblos indígenas mexicanos (véase Cuadro 3), similares a los de otros países como Australia (Furneaux y Brown, 2007) o Canadá (Gallagher y Lawrence, 2012). 


\section{Cuadro 3}

\section{CARACTERÍSTICAS DE LA POBLACIÓN OBJETO DE ESTUDIO}

\begin{tabular}{|c|c|}
\hline Población total & 103263388 \\
\hline Población indígena & 9854301 \\
\hline Porcentaje con respecto al total & $9,54 \%$ \\
\hline Grupos etnolingüísticas & 68 \\
\hline Habitantes de lengua indígena & 5988557 \\
\hline Porcentaje de analfabetismo (población de 15 años y más) & $21,6 \%$ \\
\hline Hombres & $15,7 \%$ \\
\hline Mujeres & $27,1 \%$ \\
\hline Porcentaje de inasistencia escolar (población de 6 a 14 años) & $8,4 \%$ \\
\hline \multicolumn{2}{|l|}{ Nivel de instrucción de la población indígena de 15 a 64 años* } \\
\hline Sin instrucción & $36,4 \%$ \\
\hline Primaria completa & $37,9 \%$ \\
\hline Secundaria completa & $32,4 \%$ \\
\hline Media superior & $20,5 \%$ \\
\hline Superior & $9,3 \%$ \\
\hline Población con derecho a servicios de salud & $72,8 \%$ \\
\hline Viviendas con piso de Tierra & $38 \%$ \\
\hline Viviendas con agua entubada & $69,6 \%$ \\
\hline Viviendas con drenaje & $53,5 \%$ \\
\hline \multicolumn{2}{|l|}{ Ingresos ** } \\
\hline Sin ingresos & $24,5 \%$ \\
\hline Menos de $1 * * * \operatorname{SMN} * * * *$ & $27,6 \%$ \\
\hline De 1 a 2 SMN & $28,5 \%$ \\
\hline Más de 2 SMN & $19,4 \%$ \\
\hline \multicolumn{2}{|c|}{$\begin{array}{l}\text { * Con respecto al total de la población indígena de } 15 \text { a } 64 \text { años } \\
* * \text { Información año } 2000 ; \text { no existe información disponible para el 2005, año del último Censo } \\
\text { *** } 1 \text { SMN en el año } 2000 \text { es equivalente a } 3,1 € \\
\text { **** SMN: Salario Mínimo Nacional }\end{array}$} \\
\hline
\end{tabular}

Fuente: Elaboración propia a partir de CDI (2006) y Navarrete (2008).

Sobre este particular, se ha de destacar, como puede observase en el anterior cuadro, que el grupo de población indígena en México representa cerca del 10\% de la población total del país. Además, los grupos indígenas presentan reseñables índices de marginalidad, derivada de aspectos tales como que más del $20 \%$ sean analfabetos y más del $35 \%$ se declare «sin instrucción», el $38 \%$ viva en viviendas con piso de tierra y casi la mitad de sus casas no tengan drenaje, o el hecho de que el $80 \%$ tenga unos ingresos diarios inferiores a $6 €$. Todos estos factores dificultan el acceso a oportunidades reales de obtención de rentas que no tengan que ver con la agricultura o la producción artesana. Autores como Brida et al. (2013), Juárez y Ramírez (2007) o Serrano et al. (2010), plantean el turismo y la creación de empresas ecoturísticas en comunidades deprimidas como medio de impulsar el desarrollo socioeconómico de las mismas. 
Para identificar este tipo de empresas en el ámbito mexicano se han tenido en cuenta las bases de datos de la Comisión Nacional para el Desarrollo de los Pueblos Indígenas (CDI), la Red Indígena de Turismo de México A.C. y la ONG internacional Echoway. Estas fuentes han puesto de manifiesto cómo uno de los mecanismos de apoyo más destacables para la creación de empresas ecoturísticas en zonas indígenas de México, es el Programa de Turismo Alternativo en Zonas Indígenas que lleva a cabo el CDI. Este organismo gubernamental ha apoyado a 169 proyectos, de los que sólo 85 han conseguido concluir el proceso de creación con éxito, debido a que son las únicas que cuentan con la infraestructura y la capacitación necesarias para ofrecer un servicio turístico adecuado.

Para conformar la población final objeto de estudio se ha tenido en cuenta tres criterios fundamentales: (1) que la actividad principal de la empresa sea el ecoturismo y se caracterice por los aspectos propios de dicha actividad; (2) que la empresa sea de nueva creación y que, por tanto, lleve pocos años operando en el mercado; y (3) que los fundadores de la empresa sean indígenas, es decir, que se adscriban a una de los 68 etnias indígenas registrados en México (CDI) y mantengan su lengua y pautas culturales indígenas (Hernández y Chávez, 2007). Finalmente, de las 85 empresas mencionadas con anterioridad, sólo 20 cumplían los tres requisitos. Debido al reducido tamaño poblacional, se optó por realizar el estudio sobre el total de las empresas que conforman la población, eliminando así cualquier posible error muestral.

\section{Cuadro 4 \\ FICHA TÉCNICA DE LA INVESTIGACIÓN}

\begin{tabular}{|l|l|}
\hline Características & Encuesta \\
\hline Población objetivo & $\begin{array}{l}\text { Empresas de ecoturismo de reciente creación, cuyos } \\
\text { socios fundadores son indígenas, tanto desde el punto de } \\
\text { vista lingüístico como de adscripción étnica y cultural }\end{array}$ \\
\hline Ámbito geográfico & $\begin{array}{l}\text { Poblados indígenas de México (Estados de Campeche. } \\
\text { Chiapas, Distrito Federal, Hidalgo, Michoacán, } \\
\text { Puebla, Quintana Roo, Veracruz y Yucatán) }\end{array}$ \\
\hline Ámbito temporal & Empresas creadas después del 2002 \\
\hline Unidad muestral & Empresa \\
\hline Tamaño poblacional* & 20 \\
\hline Periodo de recogida de la información & Julio 2008-marzo 2009 \\
\hline Encuestado & Socio-fundador de la empresa \\
\hline *El estudio se realizó a toda la población & \\
\hline
\end{tabular}

Fuente: Elaboración propia.

Una vez delimitada la población, se llevó a cabo un primer contacto telefónico, en el que se realizó una breve encuesta, solicitando información sobre el año de creación, el 
rango de edad y sexo del empresario, el número de trabajadores y/o socios, y la forma jurídica bajo la cual se constituyó la empresa. Dicha encuesta sirvió como toma de contacto con las distintas empresas, para sondear su predisposición a participar en nuestro estudio y para confirmar, en primer lugar, que efectivamente cumplían los requisitos previamente establecidos y, en segundo lugar, sus datos de localización y las condiciones de acceso al lugar, pues en algunos casos, éste sólo era posible a pie o en montura.

Por último, señalar que las empresas objeto de estudio se encuentran ubicadas en nueve regiones o estados diferentes, ubicados en la mitad sur de México: Campeche, Chiapas, Distrito Federal, Hidalgo, Michoacán, Puebla, Quintana Roo, Veracruz y Yucatán; por lo que el trabajo de campo, a pesar de tener que entrevistar sólo a 20 empresas, se alargó en el tiempo desde julio de 2008 hasta marzo de 2009. El Cuadro 4 muestra la ficha técnica de la investigación.

\subsection{Variables de medida}

En términos generales, la variable endógena o dependiente del modelo propuesto en la investigación es el éxito del proceso de creación de empresas ecoturísticas indígenas. En concreto, la revisión de la literatura relevante en torno a la medición del éxito de la empresa de reciente creación, vista en el apartado anterior, ha posibilitado la elección de dos indicadores objetivos concretos del resultado empresarial (véase Cuadro 5): la consolidación de la empresa, que hace referencia al número de años que la empresa lleva operando en el mercado, y su crecimiento, medido en función del ratio de variación del número de empleados (incluyendo las categorías de disminución del número de empleados respecto al número existente en el momento de la creación, mantenimiento del número de empleados respecto al número existente en el momento de la creación y crecimiento del número de empleados respecto al número existente en el momento de la creación).

Como variables exógenas o independientes (véase Cuadro 5), se han considerado dos, siguiendo a Lee et al. (2005): (1) actitud e implicación de la familia, considerando que todo miembro de la Comunidad que tenga lazos de sangre con el emprendedor, ya sean padres, hijos, tíos o primos, pertenece a la misma, y (2) la actitud e implicación de los amigos; ambas frente a la decisión de crear la empresa por parte del emprendedor, puesto que las redes próximas al mismo desarrollan un importante papel, sobre todo, el de acompañamiento y apoyo emocional durante el proceso de creación de la nueva empresa (Brüderl y Preisendörfer, 1998).

Por último, comentar que para llevar a cabo el contraste de las hipótesis propuestas previamente se ha empleado la técnica de regresión logística multinomial, siendo el software estadístico utilizado el SPSS versión 19. Los análisis de regresión resultan útiles cuando queremos explicar las variables dependientes en función de otras, denominadas explicativas, exógenas o independientes. Además, cuando una variable dependiente no es de tipo numérico, sino categórica, como ocurre en este caso, y se presupone que depende de una o más variables independientes también de tipo categórico, es adecuado utilizar los modelos de regresión logística, también conocidos como modelos de probabilidades, pues se estima la probabilidad de que un evento suceda. 


\section{Cuadro 5 \\ VARIABLES DE MEDIDA DE LA INVESTIGACIÓN}

\begin{tabular}{|c|c|c|l|}
\hline $\begin{array}{c}\text { Nombre de la } \\
\text { variable }\end{array}$ & $\begin{array}{c}\text { Escala de } \\
\text { medida }\end{array}$ & Tipo & \multicolumn{1}{c|}{ Descripción } \\
\hline Consolidación & Categórica & Endógena & $\begin{array}{l}\text { Número de años que la empresa lleva operando en el } \\
\text { mercado: } \\
2 \text { (mínimo) - 6 (máximo) }\end{array}$ \\
\hline Crecimiento & Categórica & Endógena & $\begin{array}{l}\text { Ratio de variación del número de empleados: } \\
- \text { menor número de empleados con respecto al inicio } \\
\text { mismo número de empleados con respecto al } \\
\text { inicio } \\
\text { mayor número de empleados con respecto al inicio }\end{array}$ \\
\hline $\begin{array}{c}\text { Actitud e } \\
\text { implicación de } \\
\text { la familia }\end{array}$ & Categórica & Exógena & $\begin{array}{l}\text { Actitud hacia la creación de la empresa, de los } \\
\text { miembros de la comunidad que tienen lazos de sangre } \\
\text { con el emprendedor: } \\
1 \text { (desanima completamente) - 5 (anima } \\
\text { completamente) }\end{array}$ \\
\hline $\begin{array}{c}\text { Actitud e } \\
\text { implicación de } \\
\text { los amigos }\end{array}$ & Categórica & Exógena & $\begin{array}{l}\text { Actitud hacia la creación de la empresa, de los } \\
\text { amigos del emprendedor, sean o no miembros de la } \\
\text { comunidad indígena: } \\
1 \text { (desaniman completamente) - 5 (animan } \\
\text { completamente) }\end{array}$ \\
\hline
\end{tabular}

Fuente: Elaboración propia.

\section{ANÁLISIS Y DISCUSIÓN DE RESULTADOS}

El análisis de datos se ha llevado a cabo por medio de dos modelos de regresión logísticos distintos, ambos con las mismas variables independientes, pero diferentes en cuanto a la variable endógena empleada en cada uno de ellos. De este modo, se realizó un primer modelo en el que la variable dependiente considerada fue la consolidación de la empresa, y las variables independientes, la actitud e implicación de la familia y los amigos. Asimismo, en el segundo modelo de regresión logística, la variable dependiente fue el crecimiento de la empresa, mientras que la actitud e implicación de la familia y los amigos fueron las variables independientes.

Seguidamente, el Cuadro 6 recoge el ajuste obtenido en ambos modelos. En los dos casos, los pseudo $\mathrm{R}^{2}$ ponen de manifiesto niveles de ajuste elevados. Así, mientras que la capacidad explicativa del modelo realizado considerando la consolidación de la empresa como variable endógena oscila entre el $59,3 \%$ y el $86,1 \%$, en el modelo desarrollado considerando el crecimiento de la empresa como variable endógena, la capacidad explicativa se sitúa entre el $24,6 \%$ y el $41,7 \%$.

Adicionalmente, los Cuadros 7 y 8 sintetizan los valores obtenidos de las estimaciones de los parámetros y su significación, considerando la consolidación de la empresa y el crecimiento de la empresa como dependientes, respectivamente. El análisis de los cuadros anteriores pone de manifiesto una serie de interesantes hallazgos. 


\section{Cuadro 6 \\ MEDIDAS DE AJUSTE DEL MODELO LOGÍSTICO MULTINOMIAL}

\begin{tabular}{|l|c|c|c|}
\hline \multirow{2}{*}{\multicolumn{1}{|c|}{ Variable dependiente }} & \multicolumn{3}{|c|}{ Pseudo $\mathbf{R}^{2}\left(\rho^{2}\right)$} \\
\cline { 2 - 4 } & Cox y Snell & Nagelkerke & McFadden \\
\hline Consolidación de la empresa &, 807 &, 861 &, 593 \\
\hline Crecimiento de la empresa &, 417 &, 470 &, 246 \\
\hline
\end{tabular}

Por un lado, respecto de la consolidación de la empresa (cuadro 7), la familia constituye la variable que más influencia presenta. En este sentido, el hecho de que la familia se mantenga al margen del proyecto de creación de la nueva empresa y no anime ni desanime al emprendedor, contribuye a incrementar las probabilidades de que la empresa llegue a alcanzar los cuatro años de duración. Ahora bien, si la familia anima completamente al emprendedor, incrementan las probabilidades de que la empresa llegue a alcanzar los cinco años de vida. Es decir, cuanto mayor es el grado de apoyo que proporciona la familia, mayores son las posibilidades de que la duración de la empresa se prolongue en el tiempo. De este modo, la influencia positiva que representa el apoyo total de la familia en el proyecto empresarial -y no un apoyo menor- contribuye a aumentar las posibilidades de que la empresa perviva en el tiempo un mayor número de años.

Ahora bien, en el crecimiento de la empresa de nueva creación (cuadro 8), se ha observado cómo el hecho de que la familia desanime ligeramente al emprendedor, muestre una actitud indiferente con él o incluso, lo anime pero sólo ligeramente, contribuyen a reducir las probabilidades de que se produzca un incremento en el número de empleados de la empresa. Con respecto a los amigos, el hecho de que éstos muestren una actitud de indiferencia hacia el empresario emprendedor (i.e., que ni lo animen ni lo desanimen), contribuye a incrementar las probabilidades de que la empresa aumente en número de empleados.

Estos hallazgos constituyen un indicador de la importancia clave del apoyo total de la familia en el proyecto de empresa en el contexto de los pueblos indígenas de la mitad sur de México, de forma que si este apoyo no llega a ser total, puede contribuir a disminuir las probabilidades de que incremente el número de empleados de la empresa. Sin embargo, con el apoyo de los amigos no sucede lo mismo, ya que el hecho de que los amigos se mantengan indiferentes al proyecto de empresa, contribuye a incrementar las probabilidades de que aumente el número de empleados de la empresa.

Resultados coherentes tanto con las culturas indígenas y las fuentes de poder tradicionales de las comunidades indígenas, como con las conclusiones de anteriores trabajos de investigación. Según De la Torre (2013), la Asamblea Comunitaria y los denominados principales juegan un papel fundamental en la toma de decisiones que incumben a la comunidad, que suele estar unida por lazos de sangre. La parentela, base de la organización social, está integrada por el padre, la madre, los hijos de la pareja y sus respectivas familias. La autoridad la ejerce el jefe de la familia, generalmente una persona mayor. Esta idea no es desconocida, pues durante muchos años la familia ha sido considerada en la sociedad occidental el eje vertebrador, en el que se desarrolla y mueve la persona. En el caso de los emprendedores indígenas de la mitad sur de México, este aspecto cobra mayor 


\section{Cuadro 7}

\section{CONSOLIDACIÓN DE LA EMPRESA - REDES CERCANAS AL EMPRENDEDOR}

\begin{tabular}{|c|c|c|c|}
\hline $\begin{array}{c}\text { Variable } \\
\text { dependiente } \\
\text { - Categorías: } \\
\text { Número de años } \\
\text { de operación de la } \\
\text { empresa } \\
\text { Seis años (a) }\end{array}$ & $\begin{array}{c}\text { Variables } \\
\text { independientes }\end{array}$ & $\begin{array}{l}\text { Ítems que componen las variables } \\
\text { independientes }\end{array}$ & $\begin{array}{l}\text { Parámetros } \\
\text { estimados y } \\
\text { significación }\end{array}$ \\
\hline \multirow{9}{*}{ Cinco años } & \multirow{5}{*}{$\begin{array}{c}\text { Actitud e } \\
\text { implicación de la } \\
\text { familia }\end{array}$} & La familia lo desanimó completamente & 000 \\
\hline & & La familia lo desanimó ligeramente & 39,639 \\
\hline & & La familia ni lo animó ni lo desanimo & $-1,560$ \\
\hline & & La familia lo animó ligeramente & 38,915 \\
\hline & & La familia lo animó completamente & $19,795 * * *$ \\
\hline & \multirow{4}{*}{$\begin{array}{c}\text { Actitud e } \\
\text { implicación de } \\
\text { los amigos }\end{array}$} & Sus amistades lo desanimaron ligeramente & $0(\mathrm{c})$ \\
\hline & & Sus amistades ni lo animaron ni lo desanimaron & $-18,652$ \\
\hline & & Sus amistades lo animaron ligeramente & $-19,101$ \\
\hline & & Sus amistades lo animaron completamente & $0(\mathrm{c})$ \\
\hline \multirow{9}{*}{ Cuatro años } & \multirow{5}{*}{$\begin{array}{c}\text { Actitud e } \\
\text { implicación de la } \\
\text { familia }\end{array}$} & La familia lo desanimó completamente & 20,085 \\
\hline & & La familia lo desanimó ligeramente & 17,231 \\
\hline & & La familia ni lo animó ni lo desanimo & $15,343 * * *$ \\
\hline & & La familia lo animó ligeramente & 36,256 \\
\hline & & La familia lo animó completamente & $-2,817$ \\
\hline & \multirow{4}{*}{$\begin{array}{c}\text { Actitud e } \\
\text { implicación de } \\
\text { los amigos }\end{array}$} & Sus amistades lo desanimaron ligeramente & $0(\mathrm{c})$ \\
\hline & & Sus amistades ni lo animaron ni lo desanimaron & $-36,179$ \\
\hline & & Sus amistades lo animaron ligeramente & $-16,037$ \\
\hline & & Sus amistades lo animaron completamente & $0(\mathrm{c})$ \\
\hline \multirow{9}{*}{ Dos años } & \multirow{5}{*}{$\begin{array}{c}\text { Actitud e } \\
\text { implicación de la } \\
\text { familia }\end{array}$} & La familia lo desanimó completamente & 000 \\
\hline & & La familia lo desanimó ligeramente & 16,280 \\
\hline & & La familia ni lo animó ni lo desanimo & $-3,666$ \\
\hline & & La familia lo animó ligeramente & 36,693 \\
\hline & & La familia lo animó completamente & $-2,333$ \\
\hline & \multirow{4}{*}{$\begin{array}{c}\text { Actitud e } \\
\text { implicación de } \\
\text { los amigos }\end{array}$} & Sus amistades lo desanimaron ligeramente & $0(\mathrm{c})$ \\
\hline & & Sus amistades ni lo animaron ni lo desanimaron & $-16,430$ \\
\hline & & Sus amistades lo animaron ligeramente & $-17,572$ \\
\hline & & Sus amistades lo animaron completamente & $0(\mathrm{c})$ \\
\hline
\end{tabular}

$* \mathrm{p}<, 10 ; * * \mathrm{p}<, 05 ; * * * \mathrm{p}<, 01$

(a) Categoría de referencia

(b) El programa elimina por defecto la categoría «las amistades lo desanimaron completamente»

(c) Parámetro redundante 


\section{Cuadro 8}

CRECIMIENTO DE LA EMPRESA - REDES CERCANAS AL EMPRENDEDOR

\begin{tabular}{|c|c|c|c|}
\hline $\begin{array}{c}\text { Variable } \\
\text { dependiente } \\
\text { - Categorías: } \\
\text { Crecimiento de } \\
\text { la empresa } \\
\text { Decrecimiento } \\
\text { (a) }\end{array}$ & $\begin{array}{c}\text { Variables } \\
\text { independientes }\end{array}$ & $\begin{array}{l}\text { Ítemes que componen las variables } \\
\text { independientes }\end{array}$ & $\begin{array}{l}\text { Parámetros } \\
\text { estimados y } \\
\text { significación }\end{array}$ \\
\hline \multirow{9}{*}{ Sin crecimiento } & \multirow{5}{*}{$\begin{array}{c}\text { Actitud e } \\
\text { implicación de } \\
\text { la familia }\end{array}$} & La familia lo desanimó completamente & $-19,151$ \\
\hline & & La familia lo desanimó ligeramente & $-35,937$ \\
\hline & & La familia ni lo animó ni lo desanimo & $-16,553$ \\
\hline & & La familia lo animó ligeramente & $-17,543$ \\
\hline & & La familia lo animó completamente & 000 \\
\hline & \multirow{4}{*}{$\begin{array}{c}\text { Actitud e } \\
\text { implicación de } \\
\text { los amigos }\end{array}$} & Sus amistades lo desanimaron ligeramente & $0(\mathrm{c})$ \\
\hline & & Sus amistades ni lo animaron ni lo desanimaron & 16,849 \\
\hline & & Sus amistades lo animaron ligeramente & 17,384 \\
\hline & & Sus amistades lo animaron completamente & $0(\mathrm{c})$ \\
\hline \multirow{9}{*}{ Con crecimiento } & \multirow{5}{*}{$\begin{array}{l}\text { Actitud e } \\
\text { implicación de } \\
\text { la familia }\end{array}$} & La familia lo desanimó completamente & $-19,151$ \\
\hline & & La familia lo desanimó ligeramente & $-35,643 * * *$ \\
\hline & & La familia ni lo animó ni lo desanimo & $-35,684 * * *$ \\
\hline & & La familia lo animó ligeramente & $-37,074 * * *$ \\
\hline & & La familia lo animó completamente & $-17,663$ \\
\hline & \multirow{4}{*}{$\begin{array}{c}\text { Actitud e } \\
\text { implicación de } \\
\text { los amigos }\end{array}$} & Sus amistades lo desanimaron ligeramente & $0(\mathrm{c})$ \\
\hline & & Sus amistades ni lo animaron ni lo desanimaron & $35,547 * * *$ \\
\hline & & Sus amistades lo animaron ligeramente & 35,740 \\
\hline & & Sus amistades lo animaron completamente & $0(\mathrm{c})$ \\
\hline
\end{tabular}

$* \mathrm{p}<, 10 ; * * \mathrm{p}<, 05 ; * * * \mathrm{p}<, 01$

(a) Categoría de referencia

(b) El programa elimina por defecto la categoría «las amistades lo desanimaron completamente» de la variable exógena

(c) Parámetro redundante

relevancia al supeditar la decisión de crear o no la empresa al apoyo y autorización de la Asamblea Comunitaria y del Consejo de Ancianos, lo que garantiza el apoyo de toda la comunidad a lo largo de toda la vida de la empresa (Barba y Molina, 2014).

Por otra parte, este hallazgo reafirma los resultados anteriores obtenidos en la literatura relevante en relación a la importancia clave de contar, especialmente, con el apoyo de las redes cercanas al emprendedor (e.g., Smith y Lohrke, 2008), en un contexto situacional no analizado previamente -sobre todo por las dificultades de acceso al entorno examinado-, en los procesos de creación y consolidación de empresas de nueva creación, máxime cuando los recursos de otro tipo escasean. 
En resumen, el análisis de los resultados pone de manifiesto cómo el apoyo de la familia y, especialmente, el apoyo total e incondicional de la familia, constituye un aspecto clave de las redes sociales que contribuye a incrementar las probabilidades de que el proyecto de empresa perviva un mayor número de años, así como a aumentar el número de empleados de la empresa.

\section{CONCLUSIONES Y LÍNEAS FUTURAS DE INVESTIGACIÓN}

El presente estudio se basa en la premisa de que las redes cercanas o primarias del emprendedor le ofrecen recursos, que influirán positivamente en que la empresa tenga éxito y se consolide en el mercado (Anderson et al., 2005; Lee et al., 2005). Para ello, el emprendedor debe hacer uso de los recursos incrustados en la red, es decir, del apoyo, bien sea emocional o material, que los componentes de la misma le puedan proporcionar (Brüderl y Preisendörfer, 1998).

En nuestro caso y de acuerdo con los datos que se presentan, el recurso más valioso que proporcionan las redes cercanas o primarias a los emprendedores indígenas, es el apoyo emocional. Los resultados de los modelos logísticos multinomiales aplicados, concuerdan con la teoría establecida y con los resultados esperados; aunque algunos hallazgos son peculiares, ya que en las diferentes redes cercanas se han obtenido resultados discrepantes.

En el modelo «Consolidación de la empresa», de acuerdo con los resultados, se encontró que la familia juega un rol importante, sobre todo con el apoyo emocional, ya que cada vez que la familia animaba (apoyo emocional) al emprendedor, las variables establecidas, referentes al proceso de creación de empresas, se veían afectadas de forma positiva; es decir, en el caso de que la familia proporcione apoyo emocional al emprendedor, se incrementan las posibilidades de consolidación, al lograr los 5 años de vida.

En el modelo «Crecimiento de la empresa», la familia también tiene un rol importante, sobre todo, cuando anima de forma positiva al emprendedor, ya que el crecimiento de la empresa se ve afectado positivamente gracias a dicho apoyo emocional. En el mismo modelo, pero para la variable exógena actitud e implicación de las amistades, los resultados discrepan respecto a las conclusiones obtenidas por la literatura especializada (ídem), ya que en el caso de que los amigos de los emprendedores indígenas se mantengan indiferentes, la empresa aumenta de tamaño, es decir, crece. Lo que podría explicarse por la concepción que tienen de familia (miembros de su comunidad) y amigos (miembros ajenos a la misma).

Concluyendo, el presente estudio representa un avance en las investigaciones de redes sociales, a la vez que trata el apoyo emocional proporcionado por las redes cercanas al emprendedor, en concreto, de ecoturismo indígena, temática muy poco analizada. Así, se ha confirmado las predicciones sobre la red cercana del emprendedor relativa a la familia, la cual influye de forma positiva en el proceso de creación de la empresa, siendo consistente con las conclusiones obtenidas en los trabajos de Lee et al. (2005) y de Van der Gaag y Snijders (2005). Con ello, hemos comprobado, efectivamente, que la familia de los emprendedores indígenas es una variable de influencia, principalmente de apoyo emocional, durante el proceso de creación. Respecto a la otra variable exógena, la relacionada con las amistades, éstas no tienen gran influencia en el proceso de creación, y, únicamente 
cuando son indiferentes, existe una relación positiva para que la empresa crezca. Este hecho puede entenderse si analizamos las características propias de la población objeto de estudio: las comunidades indígenas que no se han deslocalizado y todavía conservan su nexo de unión con la tierra que habitan y con sus costumbres, viven en zonas con escasa densidad de población y, a veces, de difícil acceso, aunque con un alto potencial ecoturístico, conformando un núcleo de población independiente y autónomo en el que todos sus miembros están emparentados por vínculos familiares.

El arraigo también es un factor a considerar, en consonancia con los resultados obtenidos por Wang y Altinary (2012). Para estas comunidades indígenas uno de los elementos claves es la Tierra conceptualizada como «Madre», es decir, como la proveedora de todos los recursos, por lo que cualquier acción de un individuo no solo repercute en el resto de personas y seres vivos, sino también en el medio ambiente e incluso en los seres sobrenaturales (Gallardo, 2012). Lo público se manifiesta en «mecanismos de cooperación, colaboración y reciprocidad intracomunitarios: aquellos que se dan a nivel de personas y familias, como la mano vuelta; $y$, aquellos que se dan en beneficio de la comunidad tales como el sistema de cargos, los trabajos comunitarios (tequio, faena o fajina) y los procesos de toma de decisión comunitaria a través de la asamblea comunitaria» (ibídem, 171). El apoyo de la comunidad va a suponer la implicación de sus miembros en el proyecto empresarial, bien a través de la constitución de una cooperativa o como trabajadores por cuenta ajena.

Ahora bien, el presente trabajo no está exento de ciertas limitaciones que hacen que la interpretación de los resultados haya de realizarse con cierta cautela, a la vez que dificultan la generalización de estos resultados. En efecto, el tamaño reducido de la población con el que se ha trabajado complica sustancialmente el desarrollo de planteamientos y modelos empíricos, en los que debe cuidarse especialmente el número y tipo de variables independientes que se introduzcan. Asimismo, la variable crecimiento de la empresa puede haber resultado restrictiva al haber considerado únicamente el hecho de que la empresa haya crecido, se haya mantenido estable o haya disminuido en número de empleados, sin considerar otros aspectos relacionados con la magnitud del crecimiento en el número de empleados.

Para futuras líneas de investigación, se considera interesante analizar los efectos del apoyo emocional de las redes cercanas de los emprendedores en las diferentes fases del proceso de creación de una empresa. Además, se podrían elaborar otros modelos, con diferentes variables exógenas, para medir el éxito del proceso de creación de la nueva empresa. A su vez, en posteriores estudios, se puede segmentar la investigación por grupos étnicos, para saber si existe diferencia entre los diferentes grupos indígenas mexicanos y los resultados de las redes de los emprendedores de ecoturismo indígena.

Por otro lado, los resultados obtenidos en la presente investigación, nos explican que las amistades no tienen influencia en la consolidación de la empresa, es por ello que se propone diferenciar los tipos de amistades, dependiendo de la frecuencia con que mantienen el contacto, identificando si las amistades son más o menos próximas y poder, de esta forma, encontrar otros resultados en los que podamos concluir para qué tipo de amistad tiene o no influencia el apoyo emocional proporcionado en el éxito del proceso de creación, en comparación con el apoyo de la familia. 


\section{REFERENCIAS}

ADLER, P. y KWON, S. (2002): «Social Capital: Al prospects for a new concept». Academy of Management Review, vol. 27, $\mathrm{n}^{\circ}$ 1, pp. 17-40.

ANDERSON, A.R. y JACK, S.L. (2002): «The articulation of social capital in entrepreneurial networks: A glue or a lubricant?». Entrepreneurship \& Regional Development, vol. 14, nº 3, pp. 193-210.

ANDERSON, A.R., JACK, S.L. y DODD, S.D. (2005): «The role of family members in entrepreneurial networks: beyond the boundaries of the family firm». Family Business Review, vol. 18, n 2, pp. 135-154.

ANDERSON, A.R. y MILLER, C.J. (2003): «Class matters: human and social capital in the entrepreneurial process». Journal of Socio-Economics, vol. 32, n 1, pp. 17-36.

AU, K. y KWAN, H.K. (2009): «Start-Up Capital and Chinese Entrepreneurs: The Role of Family». Entrepreneurship: Theory and Practice, vol. 33, n 4, pp. 889-908.

BARBA-SÁNCHEZ, V. y ATIENZA-SAHUQUILLO, C. (2012): «Entrepreneurial behavior: impact of motivation factors on decision to create a new venture». Investigaciones Europeas de Dirección y Economía de la Empresa, vol. 18, nº 2, pp. 132-138.

BARBA-SÁNCHEZ, V. y MOLINA-RAMÍREZ, E. (2014): «Entrepreneurship as a survival-seeking strategy for indigenous communities», en Kiril Todorov y R. Smallbone, Strategic Management in Small and Medium Enterprises: Theory and Practice. IGI Global Business Science Reference, pp. 398-420.

BARNEY, J.B. (1985): «Dimensions of informal social network structure: toward a contingency theory of informal relations in organization». Social Networks, vol. 7, pp. 1-46.

BARON, R.A. y MARKMAN, G.D. (2003): «Beyond social capital: the role of entrepreneurs' social competence in their financial success». Journal of Business Venturing, vol. 18, n ${ }^{\circ} 1,41-60$.

BECERRA, F. (2008): «Las redes empresariales y la dinámica de la empresa: aproximación teórica». INNOVAR, vol. 18, n 32, pp. 27-46.

BIRLEY, S. (1985): «The role of networks in the entrepreneurial process». Journal of Business Venturing, vol. 1, $\mathrm{n}^{\circ} 1, \mathrm{pp} .107-117$.

BLESA, A. y RIPOLLÉS, M. (2006): «Redes personales del empresario y orientación emprendedora en las nuevas empresas». Cuadernos de Economía y Dirección de la Empresa, no 26 , pp. 73-94.

BOLLINGTOFT, A. y ULHOI, J.P (2005): «The networked business incubator-leveraging entrepreneurial agency?». Journal of Business Venturing, vol. 20, $\mathrm{n}^{\circ}$ 2, pp. 265-290.

BRIDA, J.G., LANZILOTTA, B., PEREYRA, J.S. y PIZZOLOU, F. (2013): «El turismo como factor de crecimiento económico: un estudio comparado de los países del MERCOSUR». Revista de Economía Mundial, nº 34, pp. 75-96.

BRÜDERL, J. y PREISENDÖRFER, P. (1998): «Network suport and de success of newly founded businesses». Small Business Economics, vol. 10, n 3, pp. 213-225.

BURT, R.S. (2000): «The Network Structure of Social Capital». Research in Organizational Behavior, $\mathrm{n}^{\circ} 22$, pp. 345-423.

BUTLER, J.E. y HASEN, G.S. (1991): «Network Evolution, Entrepreneurial Success and Regional Development». Entrepreneurship and Regional Development, vol. 3, nº 1, pp. 1-16. 
CDI (2006): Indicadores Sociodemográficos de la Población Indígena 2000-2005. México DC., Comisión Nacional para el Desarrollo de los Pueblos Indígenas.

CHANDLER, G.N. y HANKS, S.H. (1993): «Measuring the performance of emerging businesses: a validation study». Journal of Business, vol. 8, n 5, pp. 391-408.

CHANDLER, G.N. y HANKS, S.H. (1998): «An examination of the substitutability of founders human and financial capital in emerging business venturing». Journal of Business Venturing, vol. 13, n 5, pp. 353-369.

CHELL, E. y BAINES, S. (2000): «Networking, entrepreneurship and microbusiness behavior». Entrepreneurship and Regional Development, vol. 12, n 3, pp. 195-215.

COLEMAN, J.S. (1988): «Social Capital in the creation of human capital». American Journal of Sociology, $\mathrm{n}^{\circ} 94$ (Supplement), pp. S95-S129.

COLOMBO, M.G. y GRILLI, L. (2005): «Founders' Human Capital and the growth of new tecnology-based firms: A competence-based view». Research Policy, vol. 34, $\mathrm{n}^{\circ}$ 6, pp. 795-818.

DANA, L.P. (1995): «Entrepreneurship in a Remote Sub-Artic Community: Nome, Alaska». Entrepreneurship: Theory and Practice, vol. 20, n 1, pp. 57-72.

DAVIDSSON. P. y HONIG, B. (2003): «The role of social and human capital among nascent entrepreneurs». Journal of Business Venturing, vol. 18, n 3, pp. 301-331.

DE CAROLIS, D.M. y SAPARITO, P. (2006): «Social Capital, Cognition, and Entrepreneurial Opportunities: A Theoritical Framework». Entrepreneurship: Theory and Practice, vol. 30, n 1, pp. 41-56.

DE LA TORRE, J.A. (2013): «Pluralismo jurídico y derechos humanos en la experiencia indígena mexicana de los últimos años». Revista Direito e Práxis, vol. 4, n 4, pp. 129-163.

DUBINI, P. y ALDRICH, H.E. (1991): «Personal and extended networks are central to the entrepreneurial process». Journal of Business Venturing, vol. 6, n 5, pp. 305-313.

FRANCIS, D.H. y SANDBERG, W.R. (2000): «Friendship within entrepreneurial teams and its association with team and venture performance». Entrepreneurship: Theory and Practice, vol. 25, $\mathrm{n}^{\circ} 2$, pp. 5-25.

FURNEAUX, C. y BROWN, K, (2007): «Indigenous entrepreneurship: An analysis of capital constraints». Fourth AGSE International Entrepreneurship Research Exchange, February 6-9, Brisbane (Australia).

GALLAGHER, B. y LAWRENCE, Th.B. (2012): «Entrepreneurship and indigenous identity: A study of identity work by indigenous entrepreneurs in British Columbia». International Journal of Entrepreneurship and Small Business, vol. 17, $\mathrm{n}^{\circ} 4$, pp. 395-414.

GALLARDO, E.D. (2012): «Lo público en los procesos comunitarios de los pueblos indígenas». Polis, Revista Latinoamericana, vol. 11, no 31, pp. 169-182.

GRANOVETTER, M. (1985). «Economic action and social structure: the problem of embeddedness». American Journal of Sociology, vol.91, n 3, pp.481-510.

GREVE, A. y SALAFF, J.W. (2003): «Social Networks and Entrepreneurship». Entrepreneurship Theory and Practice, vol. 28, $\mathrm{n}^{\circ}$ 1, pp. 1-22.

GULATI, R., NOHRIA, N. y ZAHEER, A. (2000): «Strategic Networks». Strategic Management Journal, vol. 21, n 3, pp. 203-215. 
HERNÁNDEZ, H.H. y CHÁVEZ, A.M. (2007): «La definición de la población indígena en el censo de población del año 2000 en México», en Hernández, H.H. (coord.). Los indios de México en el siglo XXI. Cuernavaca: UNAM, Centro Regional de Investigaciones Multidisciplinares, pp. 15-24.

HITE, J.M. y HESTERLY, W.S. (2001): «The evolution on firm networks: from emergence to early growth of the firm». Strategic Management Journal, vol. 22, no 3, pp. 275-286.

HOANG, H. y ANTONCIC, B. (2003): «Network-based research in Entrepreneurship: a critical review». Journal of Business Venturing, vol. 18, n 2, pp. 165-187.

HORMIGA, E., BATISTA, R.M. y SÁNCHEZ, A. (2007): «La influencia del capital relacional en el éxito de las empresas de nueva creación», en J.C. Ayala; Conocimiento, innovación y emprendedores: Camino al futuro. Logroño: Servicio de Publicaciones de la Universidad de la Rioja, pp. 1215.1231.

IBÁÑEZ, R.M. (2011): «Diagnóstico de la calidad y competitividad del sector turístico en México». Cuadernos de Turismo, n 28, pp. 121-143.

JOHANNISSON, B. (1996): «The dynamics of entrepreneurial networks». Frontiers of Entrepreneurship Research (Babson College, Wellesly, MA), pp. 253-267.

JOHANNISSON, B. y MONSTED, M. (1997): «Contextualizing entrepreneurial networking: The case of Scandinavia». Studies of Management \& Organization, vol. 27, $\mathrm{n}^{\circ}$ 3, 109-136.

JUÁREZ, J.P. y RAMÍREZ, B. (2007): «El turismo rural como complemento al desarrollo territorial rural en zonas indígenas de México». Scripta Nova, vol. 11, n 236, revista electrónica disponible en http://www.ub.edu/geocrit/sn/sn-236.htm.

KANTIS, H. (2004): «Un enfoque sistémico en la creación de empresas», en H. Kantis; P. Angelli y K. Morí, Desarrollo emprendedor. América Latina y la experiencia internacional. Washington, BID-FUNDES Internacional, pp. 21-34.

KIRK, R., PEREDO, A. y CHRISMAN, J. (2010): «Business Networks and Economic Development in Rural Communities in the United States». Entrepreneurship Theory and Practice, vol. 34, $\mathrm{n}^{\circ}$ 1, pp. 171-195.

KLYVER, K. y HINDLE, K. (2007): «The role of social networks at different stages of business formation». Small Business Research, vol. 15, $\mathrm{n}^{\circ}$ 1, pp. 22-38.

LARSON, A. y STARR, J.A. (1993): «A network model of organization formation». Entrepreneurship Theory and Practice, winter, pp. 5-15.

LEE, R.P.L., RUAN, D. y LAI, G. (2005): «Social structure and support networks in Bijing and Hong Kong». Social Networks, vol. 27, n 3, pp. 249-274.

LIAO, J. y WELSCH, H. (2003): «Social capital and entrepreneurial growth aspiration: a comparison of technology-and non-technology-based nascent entrepreneurs». The Journal of High Technology Management Research, $\mathrm{n}^{\circ}$ 14, pp. 149-170.

MAULA, M., AUTIO, E. y MURRAY, G. (2001): Prerequisites for the creation of Social Capital and subsequent knowledge acquisition in Corporate Venture Capital. Working Paper, Helsinki University of Technology, Helsinki (Finly).

MONSTED, M. (1995): «Processes and structures of networks: reflections on methodology». Entrepreneurial Behaviour and Research, n 7, pp. 193-213.

MORAN, P. (2005): «Structural vs. relational embeddedness: Social Capital and managerial performance». Strategic Management Journal, vol. 26, nº 12, pp. 1129-1151. 
NAHAPIET, J. y GOSHAL, S. (1998): «Social Capital, Intellectual Capital, and the organizational advantage». Academy of Management Review, vo. 23, n 2, pp. 242-266.

NAVARRETE, F. (2008): Pueblos Indígenas del México Contemporáneo. México, CDI.

NG, D. (2004). «The social dynamics of diverse and closed networks». Human Systems Management, $\mathrm{n}^{\mathrm{O}}$ 23, pp. 111-122.

OSTGAARD, T. A. y BIRLEY, S. (1994): «Personal networks and firm competitive strategy: a strategic or coincidental match?». Journal of Business Venturing, vol. 9, n 4, pp. 281-305.

PFORR, C. (2006): «Tourism policy in the making: An Austrian network study». Annals of Tourism Research, vol. 33, pp. 87-108.

SERRANO-BARQUÍN, R., PÉREZ-RAMÍREZ, C. y MANJARREZ-CAMPOS, E. (2010): «Turismo armónico como alternativa sustentable para una comunidad en el Estado de México». Estudio y Perspectivas de Turismo, nº 19, pp. 970-993.

SMITH, D.A. y LOHRKE, F.T. (2008): «Entrepreneurial network development: Trusting in the process». Journal of Business Research, $\mathrm{n}^{\circ}$ 61, pp. 315-322.

SOLYMOSSY, E. (2000). «Knowledge networks: differences and performance effects». Journal of Business Strategy, 11(1), 14-25.

STAM, W., ARZLANIAN, S. y ELFRING, T. (2014): «Social Capital of entrepreneurs and small firm performance: A meta-analysis of contextual and methodological moderators». Journal of Business Venturing, vol. 29, $\mathrm{n}^{\circ}$ 1, pp. 152-173.

SZARKA, J. (1990): «Networking and small firms». International Small Business Journal, vol. 8, n 2, pp. 10-22.

TSAI, W.M., MACMILLAN, I.C. y LOW, M.B. (1991): «Effects of strategy and environment on corporate venture success in industrial markets». Journal of Business Venturing, vol. 6, $\mathrm{n}^{\circ} 1$, pp. 9-28.

VAN DER GAAG, M. y SNIJDERS, T.A.B. (2005): «The Resource Generator: social capital quantification with concrete items». Social Networks, vol. 27, nº 1, pp. 1-29.

VAN DE VEN A.H., HUDSON, R. y SCHROEDER, D.M. (1984): «Designing new business startups: entrepreneurial organizational, and ecological considerations». Journal of Management, vol. 1, n ${ }^{\circ} 10$, pp. 87-107.

WANG, C. L. y ALTINARY, L. (2012): «Social embeddedness, entrepreneurial orientation and firm growth in ethnic minority small businesses in the UK». International Small Business Journal, vol. 30, $\mathrm{n}^{\circ}$ 1, pp. 3-23.

WITT, P. (2004): «Entrepreneurs networks and the success of start-ups». Entrepreneurship and Regional Development, vol. 16, n 5, pp. 391-412.

YOUNG, E.C. y WELSCH, H.P. (1993): «Major elements in entrepreneurial development in Central Mexico». Journal of Small Business Management, October, pp. 80-85.

ZAHRA, S.A., YAVUZ, R.I. y UCBASARAN, D. (2006): «How much do you trust me? The dark side of relational trust in new business creation in established companies». Entrepreneurship: Theory and Practice, vol. 30, nº 4, pp. 541-559. 
